\title{
Organisierte Suizidbeihilfe in Deutschland
}

\author{
Medizinische Diagnosen und persönliche Motive von 117 Suizidenten
}

F. Bruns ${ }^{1}$, S. Blumenthal ${ }^{2}$, G. Hohendorf ${ }^{3}$

Institute

1 Institut für Geschichte der Medizin und Ethik in der Medizin, Charité - Universitätsmedizin Berlin

2 Institut für Geschichte und Ethik der Medizin, Friedrich-AlexanderUniversität Erlangen-Nürnberg

3 Institut für Geschichte und Ethik der Medizin und Abteilung für Klinische Toxikologie, Klinikum rechts der Isar, Technische Universität München

\section{Zusammenfassung}

Hintergrund und Fragestellung I In der Debatte um den ärztlich assistierten Suizid spielen Ängste vor unerträglichen Leidenssituationen am Lebensende eine große Rolle. Befürworter einer Liberalisierung der ärztlichen Suizidbeihilfe verweisen regelmäßig auf Situationen, in denen Leiden auch mithilfe der Palliativmedizin nicht zu lindern sei und daher nur die Beihilfe zum Suizid einen Ausweg biete. Dabei stellt sich die Frage, ob die Fokussierung auf finale Krankheitszustände und nicht therapierbare Symptome die Realität richtig widerspiegelt.

Methodik | Wir analysierten retrospektiv Diagnosen und Motive der Menschen, die sich in Deutschland zwischen 2010 bis 2013 mit Unterstützung des Vereins „Sterbehilfe Deutschland” (StHD) das Leben genommen haben. Dafür werteten wir 118 von StHD publizierte Fallbeschreibungen aus, in denen die Suizidbegleitung sterbewilliger Vereinsmitglieder dokumentiert wird.

Ergebnisse I 67\% der Suizidenten waren über 70 Jahre alt, Frauen waren mit 71\% überrepräsentiert. Es waren folgende Diagnosen vertreten:

- 25,6\%: metastasierte Krebserkrankung

- 20,5\%: schwere neurologische Erkrankung
- 23\%: altersassoziierte Erkrankungen oder Behinderungen

- 14,5\%: psychische Erkrankung

7,7\% der Suizidenten waren körperlich und seelisch gesund.

$12,8 \%$ gaben als Hauptmotiv für ihren Suizid nicht behandelbare körperliche Symptome im Rahmen einer schweren Erkrankung an. Für 29\% war das Fehlen einer Lebensperspektive angesichts schwerer Erkrankung ausschlaggebend für die Selbsttötung. Lebensmüdigkeit ohne Vorliegen einer schweren Erkrankung gaben 20,5\% als Hauptmotiv für ihren Suizid an. Angst vor Pflegebedürftigkeit war für knapp $24 \%$ das Hauptmotiv für die Selbsttötung.

Folgerung I Unsere Untersuchung zeigt, dass sowohl die Diagnosen als auch die Beweggründe von Menschen, die organisierte Suizidbeihilfe in Anspruch genommen haben, heterogener und weniger eindeutig sind, als mitunter suggeriert wird. Unerträgliches körperliches Leiden im Endstadium einer Erkrankung spielt in diesem Zusammenhang zwar eine Rolle, andere Beweggründe überwiegen jedoch. Diese Befunde sollten bei der Debatte über die Liberalisierung der Suizidbeihilfe berücksichtigt werden.

\section{Einleitung}

In der Debatte um eine Liberalisierung des ärztlich assistierten Suizids spielen Ängste vor unerträglichen Leidenssituationen am Lebensende sowohl in den Medien als auch in der Fachöffentlichkeit eine große Rolle [1-4]. Befürworter der ärztlichen Beihilfe zum Suizid verweisen regelmäßig auf Situationen am Lebensende, in denen Leiden selbst mit den vielfältigen Therapiemöglichkeiten der Palliativmedizin nicht zu lindern sei und nur der Suizid vor einem „Qualtod“ schütze [5]. Palliativmediziner betonen demgegenüber die Möglichkeit, bei allen betroffenen Patienten eine ausreichende Symptomkontrolle zu erreichen, zumal wenn als ultima ratio die Möglichkeit der palliativen Sedierung genutzt werde [6-10].

Unabhängig von dem Dissens über die Effektivität der Palliativmedizin in besonderen Leidens- situationen [11] stellt sich die Frage, ob in der Diskussion über den ärztlich assistierten Suizid der starke Fokus auf Krankheitszustände mit absehbarem Tod („Krebs im Endstadium“ [12]) und vermeintlich nicht therapierbare körperliche Symptome das tatsächliche Spektrum der Beweggründe für den Wunsch nach Suizidassistenz richtig widerspiegelt.

In den Untersuchungen zur Praxis ausländischer Sterbehilfeorganisationen deutet sich an, dass psychosoziale Motive sehr viel häufiger zu Lebensmüdigkeit und Sterbewünschen beitragen als gemeinhin angenommen [13-16]. Ähnliches ist für Deutschland zu vermuten, wo die Datenlage jedoch begrenzt ist, da bislang einzig der Verein „Sterbehilfe Deutschland“ (StHD) eine institutionalisierte Suizidbeihilfe anbietet und Fallberichte veröffentlicht [17-20]. 
Zur Analyse der Krankheiten, körperlichen Symptome und seelischen Motive, die Menschen veranlasst haben, sich mithilfe von StHD das Leben zu nehmen, haben wir alle bislang publizierten Fallbeschreibungen dieses Vereins ausgewertet, in denen die Suizidbegleitung sterbewilliger Vereinsmitglieder in Deutschland dokumentiert wird [21-24]. Unsere Untersuchung zeigt, dass sowohl die medizinischen Diagnosen als auch die Beweggründe der Suizidenten vielschichtiger und weniger eindeutig sind, als mitunter suggeriert wird.

\section{Material und Methode}

Im Rahmen einer retrospektiven Analyse haben wir 118 Fallbeschreibungen von ärztlich assistiertem Suizid untersucht, die in den Berichtsbänden der Jahre 2011 bis 2014 des Vereins „Sterbehilfe Deutschland“ publiziert worden sind ( Tab 1). Die vier Bände dokumentieren die Fälle von ärztlicher Suizidbeihilfe durch den Verein im jeweils vorangegangenen Jahr, also im Zeitraum von 2010 bis 2013 [21-24]. Überwiegend handelt es sich um knapp gehaltene Falldarstellungen, in denen persönliche Umstände, Motive und Diagnosen der Betroffenen benannt werden. Die Darstellungen beruhen auf den Befragungen der Patienten durch die professionellen „Sterbehelfer“, darunter ein Arzt und ein Jurist.

Im Berichtsband 2012 werden die Ergebnisse der Begutachtung der Suizidwilligen durch einen Nervenarzt, der zugleich ein Vereinsmitglied ist, ausführlicher wiedergegeben [22]. In einer Fallgeschichte wird keine medizinische Diagnose erwähnt, eine weitere gibt keinen Aufschluss über Beweggründe und Motive des Suizidenten, sodass nur 117 Fälle in die Auswertung einbezogen werden konnten.

Die in den Fallgeschichten genannten medizinischen Diagnosen haben wir sechs Erkrankungskategorien zugeordnet (siehe Tab. 2). Diese haben wir in Anlehnung an eine bereits publizierte Untersuchung [19] entwickelt. Diese Untersu-

\begin{tabular}{|l|lll|}
\hline & Begleitete Suizide & Frauen & Männer \\
\hline $\mathbf{2 0 1 0}$ & 21 & 15 & 6 \\
\hline $\mathbf{2 0 1 1}$ & 27 & 21 & 6 \\
\hline $\mathbf{2 0 1 2}$ & 29 & 17 & 12 \\
\hline $\mathbf{2 0 1 3}$ & 41 & 31 & 10 \\
\hline Gesamt & 118 & 84 & 34 \\
\hline Anteil in \% & 100 & 71,2 & 28,8 \\
\hline
\end{tabular}

Tab. 1 Anzahl und Geschlecht der Personen, die von StHD zwischen 2010 und 2013 bei ihrem Suizid begleitetet wurden.

chung bezog sich auf die 27 im Berichtsband 2012 dokumentierten Fälle und ließ sich als PilotStudie für unsere größere Analyse nutzen. Bei Mehrfachnennungen medizinischer Diagnosen haben wir stets die hinsichtlich der Lebenserwartung gravierendere Erkrankung berücksichtigt.

Die in den Fallgeschichten erwähnten Beweggründe für den Suizid nahmen wir als Basis zur Erstellung von fünf Motivkategorien. Die Fallgeschichten ordneten wir daraufhin einer dieser fünf Kategorien zu. Grundlage dieser Zuordnung war das in den Schilderungen zum Ausdruck kommende Hauptmotiv für den Suizid ( $\bullet$ Tab. 3).

Alle Autoren nahmen zunächst unabhängig voneinander die Zuordnung der Fallgeschichten zu den Kategorien vor. Fälle, in denen die Autoren in ihrer Zuordnung nicht übereinstimmten, wurden in einem zweiten Schritt gemeinsam diskutiert und dann im Konsens zugeordnet. Schließlich wurden die Suizidenten verschiedenen Altersgruppen zugeordnet und nach Geschlecht unterschieden.

\section{Ergebnisse}

Diagnosen | Von 117 Personen, denen StHD zwischen 2010 und 2013 beim Suizid half, litten laut StHD-Dokumentation:

\begin{tabular}{|c|c|c|c|}
\hline & & $\begin{array}{l}\text { Anzahl } \\
\text { (absolut) }\end{array}$ & $\begin{array}{l}\text { Anteil } \\
(\%)\end{array}$ \\
\hline 1. & Fortgeschrittene Krebserkrankung mit eingeschränkter Lebenserwartung & 30 & 25,6 \\
\hline 2. & $\begin{array}{l}\text { Neurologische Erkrankung (z. B. Amyotrophe Lateralsklerose, Multiple } \\
\text { Sklerose, Apoplex, Morbus Parkinson, Paraplegie) }\end{array}$ & 24 & 20,5 \\
\hline 3. & $\begin{array}{l}\text { Herzinsuffizienz, koronare Herzerkrankung, chronisch obstruktive } \\
\text { Lungenerkrankung (COPD), periphere arterielle Verschlusskrankheit (pAVK) }\end{array}$ & 10 & 8,5 \\
\hline 4. & $\begin{array}{l}\text { Vorwiegend altersassoziierte Erkrankung (Osteoporose, Arthrose, senile } \\
\text { Makuladegeneration, Schwerhörigkeit) }\end{array}$ & 27 & 23,1 \\
\hline 5. & Ausschließlich oder vorwiegend psychische Erkrankung & 17 & 14,5 \\
\hline 6. & Altersentsprechende körperliche und seelische Gesundheit & 9 & 7,7 \\
\hline
\end{tabular}

Tab. 2 Medizinische Diagnosen der Personen, die von StHD zwischen 2010 und 2013 bei ihrem Suizid begleitetet wurden. 
- Erlösung von Schmerzen und / oder anderen körperlichen Beschwerden

- Erlebte „Sinnlosigkeit“ von Schmerzen und / oder anderen körperlichen Symptomen

2. Das Krankheitsbild nicht zu Ende aushalten wollen

- Den Angehörigen das Miterleben der Erkrankung ersparen wollen

- Eingetretener oder drohender Verlust gewohnter Fähigkeiten

3. Fehlender Lebenswille im Alter

- Einsamkeit, fehlende Kontakte

- Gefühl der eigenen Nutzlosigkeit

- Gefühl der Sinnlosigkeit des eigenen Lebens

4. Hoffnungslosigkeit, Resignation

- Depressive Selbst- und Weltsicht

- Angst, Verzweiflung

5. Drohender Verlust der Selbständigkeit

- Nicht von fremder Hilfe abhängig sein wollen

- Den Angehörigen nicht zur Lasten fallen wollen

- Nicht in ein Heim/ Hospiz wollen

Subjektiv unerträgliche körperliche

Symptome im Rahmen einer schweren

Erkrankung (z. B. Schmerzen, Dyspnoe)

Fehlende Lebensperspektive angesichts

34 schweren Erkrankung, ohne dass die unter 1. aufgeführten Symptome im Vordergrund stünden

Lebensmüdigkeit, ohne Vorliegen einer schweren Erkrankung

Aus Sicht des Suizidenten nicht behandel-
bare Symptome im Rahmen einer
psychischen Erkrankung

Angst vor Pflegebedürftigkeit 24
16
20,5

Tab. 3 Motive und Beweggründe der Suizidenten.

- $30(25,6 \%)$ an einer metastasierten Krebserkrankung,

- $24(20,5 \%)$ an einer schweren neurologischen Erkrankung

- $10(8,5 \%)$ an einer Herz-Kreislauf- oder Lungenerkrankung

- 27 (23,1\%) an altersassoziierten Erkrankungen oder Behinderungen (z. B. Osteoporose, Arthrose, vermindertes Hör- oder Sehvermögen).

- $17(14,5 \%)$ an einer psychischen Erkrankung

9 Suizidenten $(7,7 \%)$ waren körperlich und seelisch gesund ( Tab. 2).

Motive für den Sterbewunsch | 15 Suizidenten $(12,8 \%)$ gaben als Hauptmotiv für ihren Suizid nicht behandelbare körperliche Symptome im Rahmen einer schweren Erkrankung und ein daraus resultierendes unerträgliches Leiden an ( Tab. 3). Für 34 Suizidenten (29,1\%) war das Fehlen einer Lebensperspektive angesichts einer schweren Erkrankung der ausschlaggebende Beweggrund. Nicht tolerierbare körperliche Symptome, z.B. Schmerzen oder Dyspnoe, standen bei diesen Patienten nicht im Vordergrund. Lebensmüdigkeit ohne Vorliegen einer schweren Erkrankung gaben 24 Personen (20,5\%) als Hauptmotiv für ihren Suizid an. 16 Personen (13,7\%) nannten aus ihrer Sicht nicht behandelbare Symptome im Rahmen einer psychischen Erkrankung als vorherrschendes Motiv für die Selbsttötung. Die Angst vor Pflegebedürftigkeit war für 28 Suizidenten (23,9\%) das Hauptmotiv für ihren Wunsch, tot zu sein.
Alter und Geschlecht | Zwei Drittel der Menschen, die sich zwischen 2010 und 2013 mit Unterstützung durch StHD das Leben nahmen, waren 70 Jahre und älter. $71,2 \%$ der Suizidenten waren Frauen ( $\triangleright$ Tab. 1 und $\triangleright$ Tab. 4).

\section{Diskussion}

In der Debatte über die Zulässigkeit der ärztlichen Beihilfe zum Suizid wird häufig das Problem nicht behandelbarer Symptome bei Patienten mit metastasierten Karzinomen oder neurologischdegenerativen Erkrankungen in den Vordergrund gestellt. Für diese Patienten sei der ärztlich assistierte Suizid oft der letzte Ausweg aus einer unerträglichen, durch Schmerzen, Atemnot oder auch „Ekel“ geprägten Situation [25].

Abgesehen davon, dass die tatsächliche Anzahl der Fälle, in denen eine palliativmedizinische Symptomkontrolle nicht erreichbar ist, unter Experten kontrovers diskutiert wird, zeigt unsere Analyse, dass eine differenziertere Betrachtung der Diagnosen und Selbsttötungsmotive nötig ist. Die Datenlage zur ärztlichen Suizidassistenz ist in Deutschland naturgemäß schmal, da neben Einzelpersonen, die als Sterbehelfer tätig sind [26], eine institutionalisierte Suizidbeihilfe bislang einzig vom Verein „Sterbehilfe Deutschland“ angeboten wird. Unsere Studie untersucht erstmals die Erkrankungen und Motive, die die Vereinsmitglieder als Gründe für ihren Suizid angaben. 
Durch die Liberalisierung der ärztlichen Suizidassistenz würde diese Organisation, so die Erwartung mancher Autoren, „weitgehend bedeutungslos" werden [12]. Dieser Zusammenhang erscheint uns angesichts der von uns erhobenen Daten zweifelhaft. Die Tätigkeitsberichte von StHD zeigen, dass die Patienten, denen der Verein zwischen 2010 und 2013 zum Suizid verholfen hat, nicht primär an unerträglichen Schmerzen oder anderen körperlichen Symptomen litten.

Auch das Erkrankungsspektrum ist eher heterogen: Weniger als die Hälfte der Suizidenten litt an einer fortgeschrittenen Krebserkrankung oder einer schweren neurologischen Erkrankung. Ein knappes Viertel der Suizidenten litt an einer nicht-malignen altersassoziierten Erkrankung und knapp 15\% an einer psychiatrischen Erkrankung.

Weniger als 13\% der Suizidenten, die StHD bei ihrer Selbsttötung begleitet hat, gaben als Hauptmotiv für ihren Suizid subjektiv unerträgliche körperliche Symptome im Rahmen einer schweren Erkrankung an. Deutlich häufiger wurden fehlende Lebensperspektiven, Lebensmüdigkeit oder die Angst vor Pflegebedürftigkeit als Hauptmotiv genannt. Nicht behandelbare Symptome standen bei diesen Personen ausdrücklich nicht im Vordergrund.

Hervorzuheben ist aus unserer Sicht außerdem, dass über $70 \%$ der Suizidenten weiblichen Geschlechts waren - ein Anteil, der sich ähnlich auch in der Schweiz feststellen lässt [14]. Mögliche psychosoziale oder soziökonomische Gründe für dieses Ungleichgewicht wären noch zu erforschen, nicht zuletzt im Hinblick darauf, dass Frauen im Alter häufiger alleinstehend sind und meist geringere Rentenansprüche als Männer haben.

Die Tatsache, dass mehr als jeder zehnte Suizident, der sich mithilfe von StHD das Leben nahm, vorrangig an einer psychischen Erkrankung litt, bietet ebenfalls Anlass für weiteres Nachdenken. Bei depressiven Erkrankungen sowie bei Persönlichkeitsstörungen gehören Freudlosigkeit und negative Zukunftserwartungen bis hin zur Suizidalität zum Erkrankungsbild. Insofern steht die Freiverantwortlichkeit der Suizidentscheidung bei depressiven Patienten infrage.

Auch bei Persönlichkeitsstörungen, bei denen die Betroffenen oft unter chronischer Suizidalität leiden und einer langfristigen tragenden therapeutischen Beziehung bedürfen, führt „Sterbehilfe Deutschland" Suizidbegleitungen durch (vgl. [22], S. 21-30]. In den StHD-Gutachten wird die Suizidalität bei psychischen Erkrankungen häufig als krankheitsunabhängig eingestuft und der scheinbar gegebenen Selbstbestimmung der Vorrang gegenüber einer suizidpräventiven Haltung

\begin{tabular}{lll} 
Alter & Anzahl (absolut) & Anteil (\%) \\
\hline <40 Jahre & 1 & 0,8 \\
40-49 jahre & 10 & 8,5 \\
50-59 Jahre & 12 & 10,2 \\
60-69 Jahre & 16 & 13,6 \\
70-79 Jahre & 35 & 29,7 \\
80-89 Jahre & 34 & 28,8 \\
>90 Jahre & 10 & 8,5
\end{tabular}

Tab. 4 Alter der Suizidenten. und einem lebensbejahenden therapeutischen Angebot eingeräumt (beispielhaft [22], S. 18).

In neun Fällen hat StHD altersentsprechend gesunden Menschen zum Suizid verholfen. Spätestens hier wird deutlich, dass sich die Sterbehelfer, in ihrem Bestreben anderen Menschen beim Suizid zu helfen, auf einer schiefen Ebene bewegen, indem sie die ursprünglich eng gefasste „Indikation" zur Beihilfe ausweiten. Auch sind die Motive der ärztlichen Sterbehelfer zu hinterfragen, da bei altersentsprechend gesunden Suizidenten kein medizinisches, sondern ein psychosoziales Problem vorliegt.

Der starke Fokus auf nicht therapierbare körperliche Symptome, wie er in den Forderungen nach Liberalisierung ärztlicher Suizidbeihilfe häufig zu beobachten ist, spiegelt sich beim Patientenklientel von StHD nicht wider. Die in dieser Patientengruppe überwiegend anzutreffenden Leiden sind seelischer Natur und durch Krankheiten, Krisen, Lebensüberdruss oder sozial-familiäre Umstände bedingt. Damit entziehen sie sich weitgehend einer objektiven medizinischen Bewertung.

Wer wäre in der Lage, zwischen objektiv nachvollziehbarem und "nur“ subjektiv empfundenem Leiden zu gewichten und zwischen gerechtfertigter und nicht gerechtfertigter Suizidbeihilfe zu unterscheiden, zumal wenn beide Leidenssituationen von den jeweils Betroffenen als unerträglich bezeichnet werden $[1,11]$ ? Weshalb sollte der eine Zustand weniger „suizidwürdig“ sein als der andere? Wie ließen sich vor diesem Hintergrund die von Befürwortern der Suizidbeihilfe vorgeschlagenen medizinischen Sorgfaltskriterien zur Beschränkung und Kontrolle der Suizidassistenz (z.B. Vorliegen einer unheilbaren Erkrankung [25]) begründen? Dem ethischen Prinzip der Gerechtigkeit folgend wäre eine Ausweitung der Suizidbeihilfe auch auf Personen mit primär psychischem Leidensdruck naheliegend.

Gerade im Hinblick auf die mehrheitlichen Beweggründe der von StHD beim Suizid unterstützten Menschen verdienen zudem die sozialethi- 
schen Argumente gegen eine offiziell legitimierte Suizidassistenz Beachtung. So wäre etwa zu fragen, ob eine staatlich garantierte und damit normalisierte Suizidbeihilfe die häufig ambivalenten Todeswünsche vulnerabler Patienten nicht verstärken würde. Außerdem könnten nicht Sterbewillige in einen Rechtfertigungszwang getrieben werden, falls sie sich im Zustand einer womöglich kostspieligen Pflegebedürftigkeit gegen die Option eines assistierten Suizids entscheiden [27-29]. Entsprechende Befürchtungen werden durch die vorliegenden Daten aus dem USBundesstaat Oregon, wo die Praxis des ärztlich assistierten Suizids seit Jahren etabliert ist, keineswegs entkräftet [30].

Limitationen und Stärken der Studie I Die von uns analysierten Fallgeschichten geben Sichtweise und Wahrnehmung der ärztlichen und juristischen Sterbehelfer des Vereins StHD wieder. Qualität und Objektivität der Anamneseerhebung und Dokumentationspraxis konnten wir nicht überprüfen. Diese Defizite werden unseres Erachtens dadurch aufgewogen, dass wir mit unserer Studie erstmals quantifizierbare Einblicke in die Praxis der organisierten Suizidbeihilfe in Deutschland geben können.

Konsequenz für Klinik und Praxis

- In der Debatte um eine gesetzliche Regelung der Suizidbeihilfe wird unerträgliches körperliches Leiden als Argument für die Suizidassistenz angeführt. In der Praxis spielt dieses Motiv jedoch nur eine untergeordnete Rolle.

- Zu den häufigsten Beweggründen gehören eine fehlende Lebensperspektive angesichts schwerer Krankheit, Angst vor Pflegebedürftigkeit, Lebensmüdigkeit im Alter sowie psychische Erkrankungen.

- Diese Motive verweisen auf die gesellschaftliche Dimension des Problems. Ein würdiger Umgang mit Alter, Pflegebedürftigkeit und psychischen Erkrankungen beruht auf menschlicher Zuwendung, Solidarität und der politischen Bereitschaft, die nötigen finanziellen Ressourcen bereitzustellen. Eine gesetzliche Regelung zur Erleichterung der Selbsttötung sendet in diesem Zusammenhang ein sozialethisch fragwürdiges Signal.

- Eine ausgewogene Diskussion über eine mögliche Regelung der Suizidbeihilfe sollte mehr empirische Befunde zu tatsächlichen Diagnosen und Motiven berücksichtigen.

\section{Literatur}

1 Bozzaro C. Ärztlich assistierter Suizid: Kann „unerträgliches Leiden“ ein Kriterium sein? Dtsch Med Wochenschr 2015; 140: 131-134

2 Drobinski M, Hardenberg N. Nächstenliebe oder Verbrechen? Süddeutsche Zeitung vom 25.08.2014

http://www.sueddeutsche.de/politik/bundestagzu-sterbehilfe-naechstenliebe-oderverbrechen-1.2101741 Letzter Zugriff am 14.12.2015

3 Schöne-Seifert B. Wenn es ganz unerträglich wird. Frankfurter Allgemeine Zeitung vom 06.11.2014 http://www.faz.net/aktuell/feuilleton/debatten/ sterbehilfe-debatte-wenn-es-ganz-unertraeglichwird-13249733.html Letzter Zugriff am 14.12.2015

4 Nauck F, Ostgathe C, Radbruch L. Hilfe beim Sterben - keine Hilfe zum Sterben. Dtsch Arztebl 2014; 111: A-67-A-71

5 Hintze P. Kein Zwang zum Qualtod. Süddeutsche Zeitung vom 13.11.2014

http://www.sueddeutsche.de/news/leben/ gesellschaft-peter-hintze-kein-zwang-zumqualtod-dpa.urn-newsml-dpacom-20090101-141113-99-02637 Letzter Zugriff am 12.01.2016

6 Klinkhammer G. Schmerzfreiheit ist immer möglich. Dtsch Arztebl 2014; 111: A-1446-A-1448

7 Müller-Busch HC, Oduncu FS, Woskanjan S, Klaschik E. Attitudes on euthanasia, physician-assisted suicide and terminal sedation - a survey of the members of the German Association for Palliative Medicine. Med Health Care Philos 2004; 7 : 333-339

8 Carr MF, Mohr GJ. Palliative sedation as part of a continuum of palliative care. J Palliat Med 2008; 11 : 76-81

9 Schippinger W, Weixler D, Müller-Busch HC. Palliative Sedierung zur Symptomkontrolle massiver Dyspnoe. Wien Med Wochenschr 2010; 160: $338-342$

10 Sterckx S, Raus K, Mortier F, Hrsg. Continuous Sedation at the End of Life. Ethical, Clinical and Legal Perspectives. New York: Cambridge University Press; 2013

11 Bozzaro C. Der Leidensbegriff im medizinischen Kontext: Ein Problemaufriss am Beispiel der tiefen palliativen Sedierung am Lebensende. Ethik Med 2015; 27: 93-106

12 Vollmann J. Aus Respekt vor der Selbstbestimmung. Frankfurter Allgemeine Zeitung vom 20.07.2015

http://www.faz.net/aktuell/politik/die-gegenwart/ debatte-im-bundestag-ueber-sterbehilfe-indeutschland-13710439.html Letzter Zugriff am 14.12.2015

13 Bosshard G, Ulrich E, Bär W. 748 cases of suicide assisted by a Swiss right-to-die organisation. Swiss Med Wkly 2003; 133: 310-317

14 Fischer S, Huber CA, Furter M et al. Reasons why people in Switzerland seek assisted suicide: the view of patients and physicians. Swiss Med Wkly 2009; 139: 333-338

15 Flaßpöhler S. Mein Tod gehört mir. Über selbstbestimmtes Sterben. München: Pantheon; 2013

16 Oregon Health Division. Death with Dignity Act Annual Reports. Years 1-16 (1999-2014). https://public.health.oregon.gov/ ProviderPartnerResources/EvaluationResearch/ DeathwithDignityAct/Pages/ar-index.aspx Letzter Zugriff am 14.12.2015 
17 www.sterbehilfedeutschland.de Letzter Zugriff am 09.11.2015

18 Kusch R. Das letzte Recht im Leben - Wer hat die Nase vorn: Deutschland oder die Schweiz? In: Wehrli H, Sutter B, Kaufmann P, Hrsg. Der organisierte Tod. Sterbehilfe und Selbstbestimmung am Lebensende. Zürich: Orell Füssli; 2012: 57-64

19 Hohendorf G. Auf der schiefen Ebene: Zur Praxis von Sterbehilfeorganisationen in Deutschland. Z Lebensr 2014; 23: 52-57

20 Hohendorf G, Bruns F. Organisierte Suizidbeihilfe und ärztlich assistierter Suizid. Eine ethische Perspektive. Nervenheilkunde 2015; 34: 436-440

21 Kusch R, Spittler JF. Weißbuch 2011. SterbeHilfeDeutschland e. V. Norderstedt: Books on Demand; 2011

22 Kusch R, Spittler JF. Weißbuch 2012. SterbeHilfeDeutschland e. V. Norderstedt: Books on Demand; 2012

23 Kusch R, Spittler JF. Der Ausklang. Leitfaden für Selbstbestimmung am Lebensende. Norderstedt: Books on Demand; 2013

24 Kusch R, Spittler JF. Der Ausklang. Leitfaden für Selbstbestimmung am Lebensende. Norderstedt: Books on Demand; 2014
25 Hintze P, Reimann C, Lauterbach K et al. Entwurf eines Gesetzes zur Regelung der ärztlich begleiteten Lebensbeendigung (Suizidhilfegesetz) vom 30.06.2015, Bundestagsdrucksache 18/5374

26 Arnold UC. Letzte Hilfe. Ein Plädoyer für das selbstbestimmte Sterben. Reinbek bei Hamburg: Rowohlt; 2014

27 Bruns F, Hohendorf G. Contra organisierte Suizidbeihilfe. Ethik Med 2015; 27: 167-170

28 Wunder M. Ärztliche Suizidbeihilfe - das ethische Dilemma der Assistenz. In: Anderheiden M, Eckart WU, Hrsg. Handbuch Sterben und Menschenwürde. Band 2. Berlin / Boston: de Gruyter; 2012: 1055-1074

29 Spaemann R, Hohendorf G, Oduncu FS. Vom guten Sterben. Warum es keinen assistierten Tod geben darf. Freiburg: Herder; 2015

30 Finlay IG, George R. Legal physician-assisted suicide in Oregon and The Netherlands: evidence concerning the impact on patients in vulnerable groups - another perspective on Oregon's data. J Med Ethics 2011; 37: 171-174
Interessenkonflikt

Die Autoren geben an, dass kein Interessenkonflikt besteht.

DOI 10.1055/s-0041-111182

Dtsch Med Wochenschr 2016; 141: e32-e37

(c) Georg Thieme Verlag KG . Stuttgart - New York . ISSN 0012-0472 